\title{
A STUDY OF ACUTE ST ELEVATION MYOCARDIAL INFARCTION (STEMI) IN ELDERLY PATIENTS IN A TERTIARY CARE INSTITUTE IN SOUTH INDIA
}

\author{
Gopalakrishnan Senthilkumar' ${ }^{1}$ Alagarsamy Srinivasan ${ }^{2}$
}

1 Professor and HOD, Department of Cardiology, Thanjavur Medical College, Thanjavur, Tamilnadu, India.

${ }^{2}$ Senior Assistant Professor, Department of Cardiology, Thanjavur Medical College, Thanjavur, Tamilnadu, India.

\begin{abstract}
BACKGROUND
ABSTRACT

Coronary Artery Disease (CAD) is the leading cause of morbidity and mortality among elderly. The clinical features of Acute ST Elevation Myocardial Infarction (STEMI) in elderly are different from those in young patients in many aspects. The elderly STEMI patients are often less aggressively treated than younger ones due to advanced age, co-morbidities and delayed admission to the hospital. The epidemiological data regarding STEMI in elderly in India are mainly from urban centres.

Hence, we conducted a study on the clinical presentation, risk factors and complications of elderly STEMI (> 60 years) patients admitted in the ICCU of Department of Cardiology, Thanjavur Medical College, Tamilnadu which caters to the needs of largely rural population.
\end{abstract}

\section{MATERIALS AND METHODS}

Our retrospective descriptive study was conducted on patients $>60$ years of age, who were admitted with the features of acute STEMI from $1^{\text {st } J a n u a r y ~} 2018$ to $30^{\text {th }}$ September 2018. The demographic features, cardiovascular risk factors, clinical presentations, serial ECG changes and 2D-Echo features were analysed and correlated.

\section{RESULTS}

Out of 87 patients studied, 58 (66.67\%) were Male and 29 (33.33\%) were Female with Male-to-Female ratio of 2:1. However, above 80 years of age, there were more Females (20.69\%) than Males (1.72\%). Majority of the patients belonged to 60-70 years' age group (66.67\%). Type II Diabetes Mellitus (28.74\%) and Systemic Hypertension (25.29\%) were the common risk factors. Though 50.57\% of patients presented with chest pain, atypical symptoms were present in significant number of patients. Majority were in Killip's class II (47.13\%) on admission. IWMI occurred slightly more (49.42\%) than AWMI (48.28\%). RVMI occurred in 37.21\% of patients with acute IWMI. LVEF < 40\% was more common in AWMI (64.28\%) group and in non-thrombolysed (50\%) patients. Mitral regurgitation was present in 57 (65.52\%) cases, while cardiogenic shock was present in $14.95 \%$ of cases. 5 patients (5.74\%) died during their hospital stay.

\section{CONCLUSION}

STEMI in the elderly presented commonly between 60-70 years of age. Female patients with STEMI increased with increasing age ( $>70$ yrs.). Diabetes Mellitus and Systemic Hypertension were the common risk factors. More patients presented with atypical symptoms and in Killip's class II. IWMI was more common than AWMI. 37.21\% of patients with IWMI had RVMI. LV systolic dysfunction with LVEF < 40\% was more common in AWMI and in non-thrombolysed patients. Advanced age, female gender, multiple risk factors, co-morbid conditions, extensive infarction and severe LV systolic dysfunction were the major contributors of in-hospital mortality.

\section{KEY WORDS}

STEMI in Elderly, LV Dysfunction, Thrombolysis, Cardiogenic Shock.

HOW TO CITE THIS ARTICLE: Senthilkumar G, Srinivasan A. A study of acute ST elevation myocardial infarction (STEMI) in elderly patients in a tertiary care institute in South India. J. Evolution Med. Dent. Sci. 2018;7(49):5219-5224, DOI: $10.14260 /$ jemds/2018/1158

\section{BACKGROUND}

Coronary artery disease remains the leading cause of morbidity and mortality among elderly patients worldwide. ${ }^{1}$ Acute Coronary Syndromes (ACS) include Unstable Angina, Non-ST Elevation Myocardial Infarction (NSTEMI) and ST Elevation Myocardial Infarction (STEMI). ${ }^{2}$ The incidence of ACS and the prevalence of ST Elevation Myocardial Infarction progressively increases with age. ${ }^{3}$

'Financial or Other Competing Interest': None.

Submission 09-11-2018, Peer Review 22-11-2018,

Acceptance 24-11-2018, Published 03-12-2018.

Corresponding Author:

Dr. Alagarsamy Srinivasan,

HIG 419, Tamil University Colony,

Pillaiyarpatti, Thanjavur-613010, Tamilnadu, India.

E-mail: apcardiotmch@gmail.com

DOI:10.14260/jemds/2018/1158

STEMI is associated with significantly higher mortality in the elderly when compared with the young. Yet the elderly people are less aggressively treated than the young. ${ }^{4}$ Thrombolytic therapy has the greatest effect in the elderly, even though there is increased risk of haemorrhagic stroke. ${ }^{5}$

The clinical features of STEMI in the elderly are different from the young in many aspects. ${ }^{6,7}$ Elderly STEMI patients present with more atypical symptoms and co-morbidities than the younger ones. 89 In India, the studies regarding the elderly STEMI patients were mostly from urban centres. ${ }^{10,11}$ Hence, we studied the clinical, ECG and Echocardiographic profile of elderly STEMI patients ( $>60$ years of age) admitted in Thanjavur Medical College, Thanjavur which is a tertiary referral centre for nearly 5 adjacent districts in and around Thanjavur with largely Rural and Agricultural population. 
The risk stratification of STEMI at the time of admission and at discharge from the hospital by clinical, ECG and Echocardiographic parameters accurately determine the outcome of patients with STEMI and helps in determining the Optimal Therapeutic Interventions and secondary preventive measures.

\section{MATERIALS AND METHODS}

Our retrospective descriptive study was conducted on patients above 60 years of age who were admitted with the features of acute ST Elevation Myocardial Infarction (STEMI) in the ICCU of Department of Cardiology, Thanjavur Medical College, Thanjavur, India from $1^{\text {st }}$ January 2018 to $30^{\text {th }}$ September 2018.

The patients satisfying the following inclusion criteria were included in the study.

\section{Inclusion Criteria}

- Age- 60 years (or) above (ICMR Guidelines).

- Typical ECG pattern.

a. ST segment elevation of $>0.1 \mathrm{mv}$ in at least two consecutive limb leads (or) $>0.2 \mathrm{mv}$ in at least two consecutive chest leads.

b. Definite ' $\mathrm{T}$ ' wave inversion and pathological evolution of $\mathrm{Q}$ waves ( $>0.04 \mathrm{sec}$ ).

c. New onset of LBBB.

- $\quad$ Elevated cardiac enzyme levels (Troponin T).

The clinical data studied include Demographic features like diabetes mellitus, systemic hypertension, dyslipidaemia, tobacco smoking/chewing and alcohol consumption.

The presenting symptoms studied include chest pain, shortness of breath, sweating, palpitation, dizziness, syncope, nausea/vomiting and abdominal pain. The time of presentation of patient to the I.C.C.U from the onset of symptoms (window period) and the Killip Clinical Class of the patient were analysed.

The serial electrocardiograms (E.C.G) recorded by the computerized 12 channel E.C.G machine (BIONET) were analysed for ST segment elevation (or) depression, pathological Q waves, tachy/bradyarrhythmias and conduction disturbances. The regions of infarction were categorized into Anterior, Inferior (or) Combined using standard AHA E.C.G criteria.

Echocardiography was performed on all patients with STEMI by consultant cardiologists using Philips HD11XE and Aloka Prosound SSD 4000 Echocardiography systems within 24 hours of admission of the patient to I.C.C.U and at the time of discharge. The data studied include Regional Wall Motion Abnormality suggestive of ischemia (RWMA), LV and RV chamber dimensions, LV ejection fraction, mitral regurgitation, LV clot, pulmonary hypertension, tricuspid regurgitation, RV function, pericardial effusion, identification of ventricular septal rupture and ventricular aneurysm.

\section{RESULTS}

A total of 87 elderly patients ( $>60$ years of age) admitted with the features of Acute Elevation Myocardial Infarction (STEMI) in the ICCU of Department of Cardiology at Thanjavur Medical College, Thanjavur from 1st January 2018 to $30^{\text {th }}$ September
2018 were studied retrospectively regardless of the therapeutic intervention they received.

Out of the 87 patients, 58 (66.67\%) were male and 29 $(33.33 \%)$ patients were female with the male-to-female ratio of $2: 1$. The common age group of presentation in the elderly was between $60-70$ years $(66.67 \%)$ of age. In the $>80$ years' age group, there were more female $(29.69 \%)$ than male $(1.72 \%)$ in our study, (Figure 1).

Type II Diabetes mellitus was the most common risk factor (28.74\%) followed by systemic hypertension (25.29\%) among overall elderly patients with STEMI (Figure 2). Diabetes mellitus is slightly more common in elderly female (31.03\%) than elderly male $(27.59 \%)$ patients with STEMI.

Chest pain was the most common presenting symptom of STEMI (50.57\%) followed by atypical symptoms like difficulty in breathing (37.93\%), sweating, nausea (26.44\%), vomiting $(25.29 \%)$ and dizziness $(16.09 \%)$ in elderly patients. Around $49.43 \%$ of patients presented to the ICCU within 12 hours of onset of chest pain, while $33.33 \%$ between $12-24 \mathrm{hrs}$. and $17.24 \%>24$ hours after the onset of symptoms. Killip's class II was the most common (47.13\%) clinical class followed by Killip's class I (25.29\%), class IV (14.97\%) and class III $(12.64 \%)$ in our study. Co-morbid conditions like renal insufficiency was present in $10(11.50 \%)$ cases, while old CVA was present in 3 (3.45\%) cases.

The ECG localisation of infarction showed inferior wall MI $(49.42 \%)$ occurred slightly more than anterior wall MI $(48.28 \%)$ in the elderly STEMI patients. 2 patients $(2.30 \%)$ had both anterior and inferior wall MI. Among the 43 patients (49.42\%) with IWMI, inferoposterior wall MI is more common $(32.86 \%)$. RVMI occurred in $37.21 \%$ of elderly STEMI patients with acute IWMI in our study, (Figure 3). Out of the 42 elderly patients (48.28\%) with Acute AWMI, 19 (45.24\%) had anteroseptal MI and 18 (42.86\%) had extensive anterior wall MI. 3 cases $(7.14 \%)$ had anterolateral MI, while 2 cases (4.76\%) had high lateral MI (Figure 4).

The 2D Echocardiographic evaluation done within 24 hours of acute STEMI showed regional wall motion abnormality suggestive of anterior wall ischaemia in the form of hypokinesia $(80.95 \%)$ and akinesia $(11.90 \%)$ in patients with acute AWMI. Similarly, in patients with acute IWMI, $81.40 \%$ had hypokinesia and $6.98 \%$ had akinesia as RWMA.

The 2D Echocardiographic study of LV systolic function by the measurement of LVEF showed $64.28 \%$ of patients with acute AWMI had moderate-to-severe LV systolic function with $\mathrm{EF}<40 \%$. The LVEF was normal in $2.38 \%$ of cases. The assessment of LV systolic function in elderly patients with IWMI showed only $11.63 \%$ patients had moderate-to-severe LV systolic function with LVEF $<40 \%$. While $62.79 \%$ had mild LV systolic function with LVEF between 40-55\%. The LVEF was normal in $25.58 \%$ of cases (Figure 5). The mitral regurgitation was present in 57 (65.52\%) of elderly STEMI patients. Out of these 57 patients, 31 (35.63\%) cases had IWMI and 26 cases $(29.89 \%)$ had AWMI.

Out of the 87 patients with STEMI 53 (60.92\%) cases were thrombolysed, while $34 \quad(39.08 \%)$ cases were not thrombolysed. Advanced age and late presentation to the hospital were the main reasons for not receiving Thrombolysis in these patients. Nearly, $50 \%$ of patients who were not thrombolysed had moderate-to-severe LV systolic dysfunction 
with LVEF $<40 \%$, while only $32.08 \%$ of patients who received thrombolysis had $\mathrm{EF}<40 \%$ (Figure.6).

Out of the 87 patients, $5(5.74 \%)$ patients died during their hospital stay. All of them were above 65 years of age. Among them 4 were female and 1 was a male patient. 3 cases were thrombolysed, while 2 cases did not receive Thrombolysis. 4 patients had inferoposterior and RVMI, while one had extensive AWMI. Cardiogenic shock was the cause of death in 3 cases, while Ventricular fibrillation and cardiac asystole contributed to the death in each of the remaining 2 cases.

\section{STEMI in Elderly-Figures}
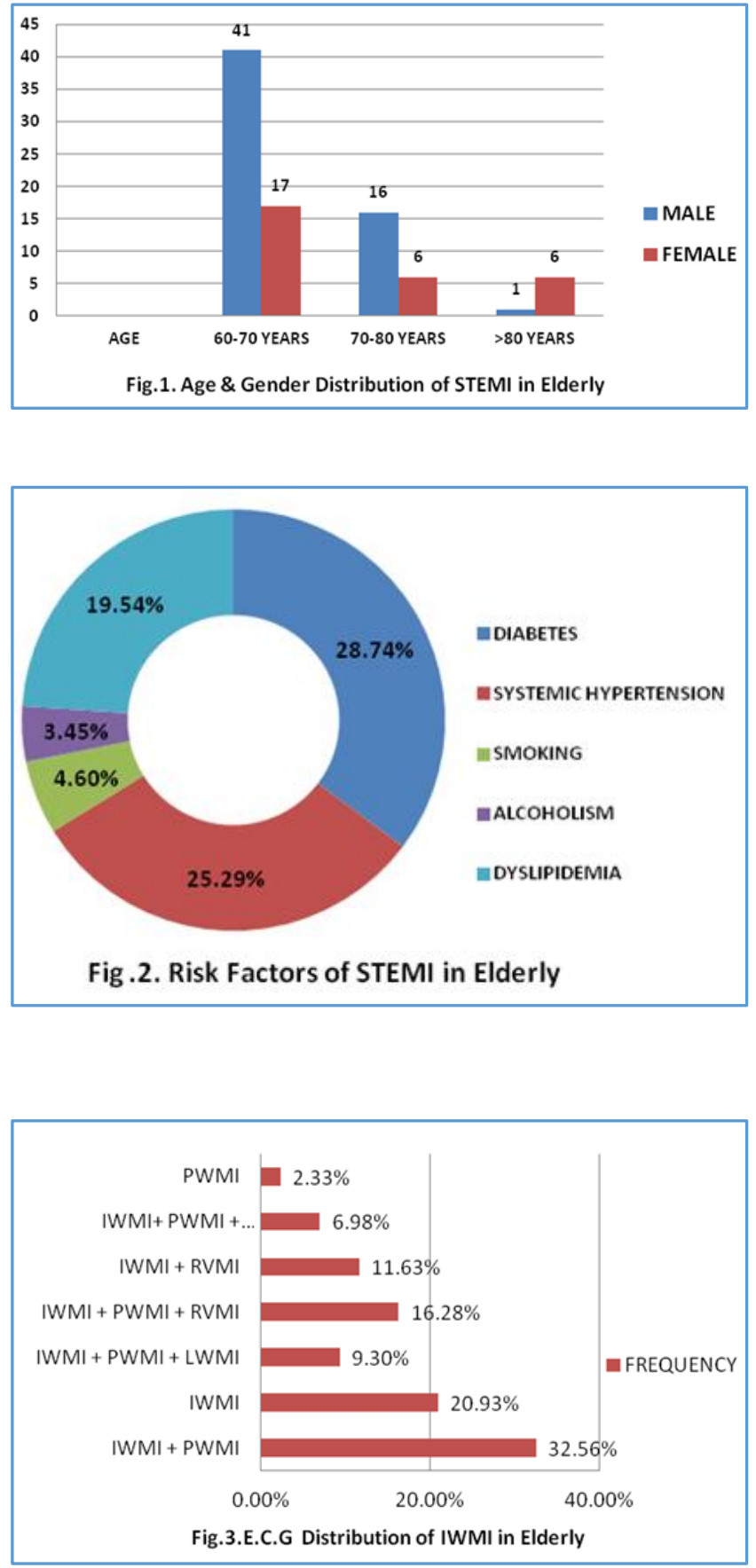
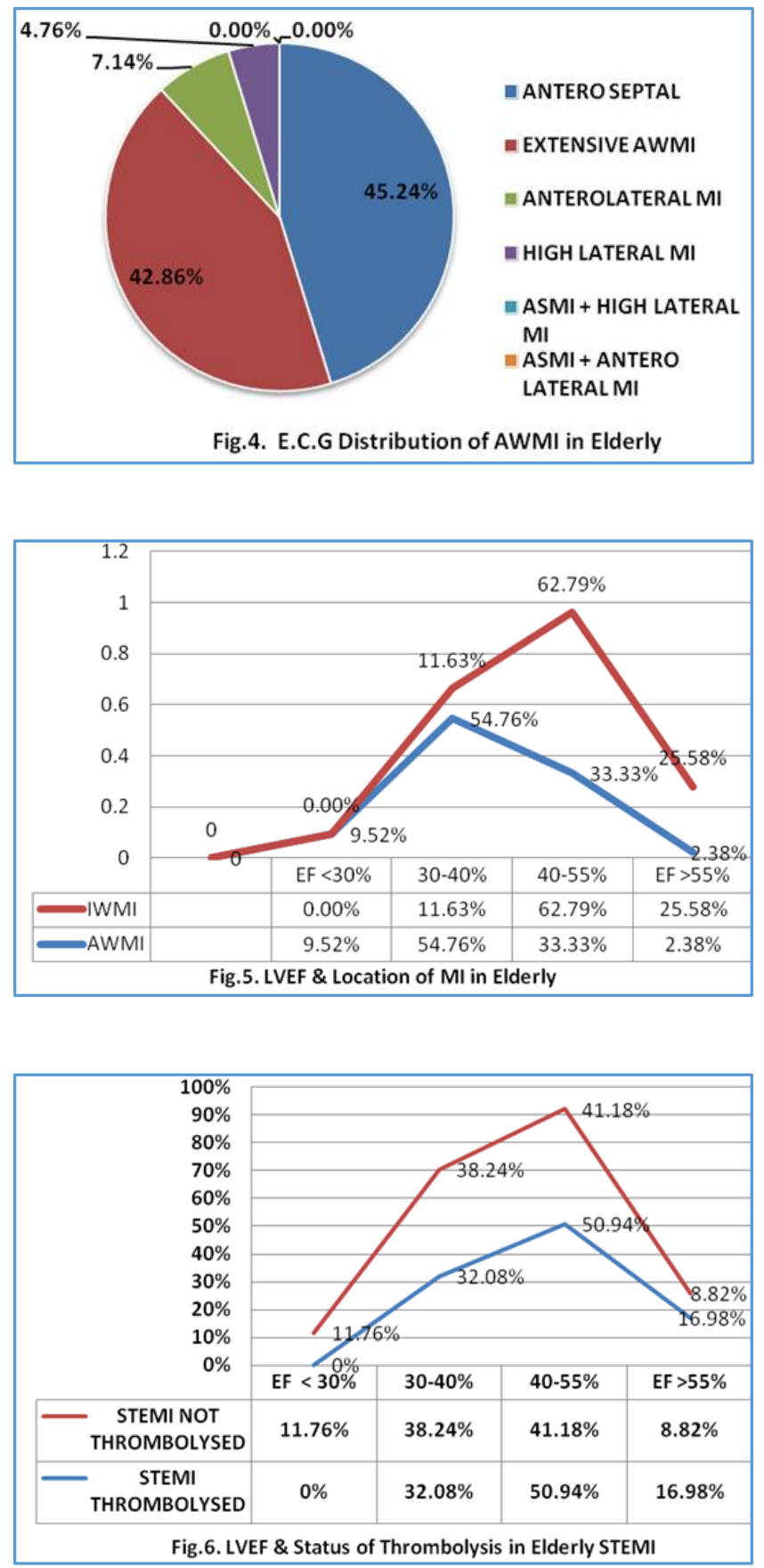

\section{DISCUSSION}

The Coronary Artery Disease is the leading cause of death among elderly patients. The Elderly patients with Acute ST Elevation Myocardial Infarction (STEMI) often present with more atypical symptoms.12 Though, STEMI in elderly is associated with significantly higher mortality. They are treated less aggressively than the young. ${ }^{13}$

Majority of elderly STEMI patients belonged to 60-70 years of age group in the present study. This is in concordance to other studies, wherein the number of elderly presenting with STEMI decreases as age increases. This is attributed to the comorbid condition like renal insufficiency, cognitive problems, depression and atypical vague symptoms of MI in elderly forbidding them proper access to health care. ${ }^{1}$ 
In the present study, $66.67 \%$ of elderly patients were male and $33.33 \%$ were female with the male-to-female ratio of 2:1. However, above the age of 80 years there were more female (20.69\%) than male (1.72) in the present study. This indicates an increase in prevalence of disease in elderly females compared to young females who are hormonally protected against CAD. Alexander $\mathrm{K}$ et al reported that with progressively older age, patients with ACS are more likely to be female from $30 \%$ below age 65 to $62 \%$ over the age of 85 years. ${ }^{14}$ This gender and CV risk reverses past age 65 . In a statistical study by AHA, the prevalence of cardiovascular diseases increased in female as the age increases (male-tofemale ratio of 1.3:1 in 35-44 years of age group compared to 0.89:1 in 75+ age group). ${ }^{15}$ The loss of protective effect of oestrogen in postmenopausal woman may contribute to the increased incidence of STEMI in them with advanced age.

Though chest pain was the common presenting symptom of STEMI (50.57\%) in Elderly patients, atypical symptoms like difficulty in breathing (37.93\%), nausea, vomiting and dizziness were also significantly present in elderly STEMI patients. In Worcester Heart Attack Study chest pain was reported in less than half of the patients over age 75 years (45.5\%), while dyspnoea (or) cough (22\%) and other symptoms like dizziness, headache, syncope, sweating, palpitation, nausea and weakness (32\%) were more common. 16

In the present study, Type II Diabetes mellitus was the most common risk factor followed by Systemic Hypertension in elderly STEMI patients. The incidence of Type 2DM is slightly more in elderly female than in male.

The smoking and alcoholism contributed to the risk factors of STEMI in only a few elderly STEMI patients in the present study. Various studies have reported that hypertension, diabetes mellitus and dyslipidaemia were just as prevalent in both young and elderly STEMI patients while smoking, obesity and family history of CAD are more prevalent in the young STEMI patients. ${ }^{13}$ The emphasis for the elderly population should be better control of diabetes, systemic hypertension and dyslipidaemia, while in young population in addition to quitting smoking habits control of obesity should be included. Only $49.43 \%$ of patients presented to the ICCU within 12 hours of onset of symptoms in the present study. Atypical clinical presentation might have caused pre-hospital delay in the admission of elderly STEMI patients. In the Global Registry of Acute Coronary Events (GRACE) registry, the median time from symptoms onset to presentation was 2.3 hours in those under 45 years, but 3 hours over age $85 .{ }^{1}$ In the Co-operative Cardiovascular Project, one significant determinant of late arrival ( $>6$ hours of symptom onset) was advanced age.

The Inferior wall MI occurred slightly more $(49.42 \%)$ than Anterior wall MI (48.28\%) in our study. Various studies reported that in patients $<60$ years of age, AWMI is more common than IWMI. Teruo Shiraki et al reported AWMI was present in $52 \%$ of patients in the above 70 years' age group. ${ }^{17}$ Among the patients with AWMI 48.28\% had anteroseptal MI, while $45.24 \%$ had extensive anterior wall MI in our study. Various studies have reported that patients with AWMI have worse prognosis with increased incidence of complications than IWMI and the occurrence of acute RVMI with IWMI have worse prognosis than Acute Inferior Wall MI alone.18 Right Ventricular Infarction (RVMI) occurred in $37.21 \%$ of elderly STEMI patients with Acute IWMI in the present study.
Most of the in-hospital mortality in STEMI depends on the severity of LV systolic dysfunction. The risk of sudden cardiac death after MI is increased by the development of post infarct heart failure. ${ }^{19}$ The 2D Echocardiography done within 24 hours of admission can predict the patients with high risk, diagnose mechanical complications of STEMI and quantity of the LV dysfunction. ${ }^{20}$ Thus, it aids in the treatment and assessing the prognosis of patients with acute STEMI. Various studies have shown that Echo findings in STEMI correlates well with the clinical events and ECG in AMI.21,22

In our study out of 43 patients with IWMI, Regional Wall Motion Abnormality (RWMA) suggestive of ischaemia was observed in the form of Hypokinesia (81.40\%) and Akinesia (6.98\%) in the corresponding segments of LV by 2D Echo. Similarly, among 42 patients with AWMI 80.95\% had hypokinesia and $11.90 \%$ had akinesia as RWMA.

There were more patients (64.28\%) with moderate-tosevere LV systolic dysfunction with LVEF $<40 \%$ in elderly AWMI group than in IWMI group (11.63\%) in the present study. The LVEF was normal in $25.58 \%$ of IWMI cases, while only $2.38 \%$ of AWMI cases had normal LV systolic function. A left Ventricular Ejection Fraction (LVEF) less than $40 \%$ was an independent mortality predictor in Multicenter Post Infarction Research Group in the 1980s. ${ }^{23}$ The Canadian Assessment of Myocardial Infarction (CAMI) study also found LVEF $<40 \%$ appears to be responsible for an increase in post MI risk. ${ }^{24}$

In the present study $60.92 \%$ of cases were thrombolysed, while $39.08 \%$ of cases were not thrombolysed. Nearly, $50 \%$ of cases who were not thrombolysed had LVEF $<40 \%$, while only $32.08 \%$ had LVEF $<40 \%$ in the thrombolysed group in the present study. The main reasons for not thrombolysing these patients were advanced age, delayed presentation to the hospital and co-morbid conditions. In the Grace Registry, 30\% of STEMI patients presenting within 12 hours of symptoms did not receive thrombolytic therapy. Older age ( $>75$ years), female sex, absence of chest pain and congestive cardiac failure were the factors associated with failure to receive thrombolysis in STEMI patients in this Registry.

Mitral regurgitation was present in 57 (65.52\%) of elderly STEMI patients in our study. Various studies have shown that MR develops in 14-26\% of patients with STEMI. Cardiogenic shock was present in $14.95 \%$ of cases in our study. Ornato JP et al in their study about factors associated with the occurrence of cardiac arrest during hospitalisation for AMI reported that cardiogenic shock occurs in $>10 \%$ of patients > 75 years of age mostly due to ventricular (or) papillary muscle rupture (or) severe LV (or) RV dysfunction.

5 patients (5.74\%) died during their hospital stay in our study. All of them were $>65$ years of age with 4 female and 1 male. 4 had inferoposterior and RVMI, while one had extensive AWMI. Cardiogenic shock was the cause of death in 3 cases, while ventricular fibrillation and cardiac asystole contributed to the death in each of the remaining 2 cases.

In a comparative study between elderly and young MI, mortality was on the higher side in the elderly group. In PURSUIT trial among STEMI patients, in-hospital mortality increased exponentially as a function of age from $1.9 \%$ among patients age $<40$ years to $31.9 \%$ among patients older than 80 years. In GUSTO-1 trial, 30-day mortality following STEMI increased from $3 \%$ in patients < 65 years of age to $19.6 \%$ in patients 75 to 85 years of age and to $30.3 \%$ in patients $>85$ years of age. Age, Gender and LV dysfunction are the most 
powerful predictors of in-hospital and 30day mortality in various studies. ${ }^{25,26}$

\section{Limitations}

Our analytical study reflects a single tertiary care institute retrospective observation. It is not a population-based study. This may result in several bias. The clinical follow-up details of the patients were not available. Though, ECG and Echocardiography are important for diagnosis and management of STEMI, Gold standard coronary angiography was not done in our study.

\section{CONCLUSION}

Majority of the elderly STEMI patients belonged to 60-70 years' age group and were male. However, the number of female patients with STEMI increased with increasing age. Above the age of 80 years, there were more Female than Male patients with STEMI. Although, the chest pain was the commonest presenting symptom, atypical presentations were seen in significant number of cases which included dyspnoea, nausea, vomiting and dizziness. Conventional risk factors like Type II Diabetes Mellitus and Systemic Hypertension were commonly present in both genders in the elderly. Most of the patients presented between 6-12 hours after the onset of symptom. $39 \%$ of cases did not receive Thrombolysis. Advanced age and delayed presentation to the hospital were the main reasons for not thrombolysing them. Inferior wall MI was slightly more common than AWMI. RVMI occurred in $37.21 \%$ of elderly STEMI patients with Acute IWMI. LV systolic dysfunction with increasing severity was observed in AWMI than in IWMI. Patients who did not receive thrombolysis also showed increased severity of LV systolic dysfunction. Ischaemic mitral regurgitation was present in $65.52 \%$ of elderly STEMI patients. Advanced age, female gender, multiple risk factors, co-morbid conditions, extensive infarction and severe LV systolic dysfunction were the major contributors of Hospital Mortality in elderly Acute STEMI patients.

\section{REFERENCES}

[1] Avezum A, Makdisse M, Spencer F, et al. Impact of age on management and outcome of acute coronary syndrome:observations from the Global Registry of Acute Coronary Events (GRACE). Am Heart J 2005;149(1):67-73.

[2] Myocardial infarction redefined-a consensus document of The Joint European Society of Cardiology / American College of Cardiology Committee for the redefinition of myocardial infarction. Eur Heart J 2000;21(18):150213.

[3] Mehta RH, Rathore SS, Radford MJ, et al. Acute myocardial infarction in the elderly:differences by age. J Am Coll Cardiol 2001;38(3):736-41.

[4] Smith SC Jr. Drug treatment after myocardial infarction; is treatment the same for the elderly as in the young patient? Am J Geriatr Cardiol 1998;7(1):60-4.

[5] Eagle KA, Goodman SG, Avezum A, et al. Practice variation and missed opportunities for reperfusion in ST-segment-elevation myocardial infarction:findings from the Global Registry of Acute Coronary Events (GRACE). Lancet 2002;359(9304):373-7.

[6] Day JJ, Bayer AJ, Pathy MS, et al. Acute myocardial infarction:diagnostic difficulties and outcome in advanced old age. Age Ageing 1987;16(4):239-43.

[7] Yang XS, Willems JL, Pardaens J, et al. Acute myocardial infarction in the very elderly. A comparison with younger age groups. Acta Cardiol 1987;42(1):59-68.

[8] Tresch DD. Management of the older patient with acute myocardial infarction:difference in clinical presentation between older and younger patients. J Am Geriatr Soc 1998;46(9):1157-62.

[9] Ochiai ME, Lopes NH, Buzo CG, et al. Atypical manifestation of myocardial ischemia in the elderly. Arq Bra Cardiol 2014;102(3):e31-3.

[10] Sandhya S, Mohanraj P. Clinical presentation, risk factors, complications and outcome of acute myocardial infraction in elderly patients. Int J Res Med Sci 2017;5(11):4765-9.

[11] Bhatia LC, Naik RH. Clinical profile of acute myocardial infarction in elderly patients. J Cardiovasc Dis Res 2013;4(2):107-11.

[12] Tresch DD, Brady WJ, Aufderheide TP, et al. Comparison of elderly and younger patients with outof-hospital chest pain. Clinical characteristics, acute myocardial infarction, therapy and outcomes. Arch Intern Med 1996;156(10):1089-93.

[13] Paul SD, O'Gara PT, Mahjoub ZA, et al. Geriatric patients with acute myocardial infarction:cardiac risk factor profiles, presentation, thrombolysis, coronary interventions and prognosis. Am Heart J 1996;131(4):710-5.

[14] Alexander KP, Roe MT, Chen AY, et al. Evolution of cardiovascular care for elderly patients with non-STsegment elevation acute coronary syndromes:results from CRUSADE. National Quality Improvement Initiative. J Am Coll Cardiol 2005;46(8):1479-87.

[15] Mikkola TS, Gissler M, Merikukka M, et al. Sex differences in age-related cardiovascular mortality. PLoS One 2013;8(5):e63347.

[16] Saczynski JS, Yarzebski J, Lessard D, et al. Trends in prehospital delay in patients with acute myocardial infarction (from the Worcester Heart Attack Study). Am J Cardiol 2008;102(12):1589-94.

[17] Shiraki T, Saito D. Clinical features of acute myocardial infarction in elderly patients. Acta Med Okayama 2011;65(6):379-85.

[18] Stone PH, Raabe DS, Jaffe AS, et al. Prognostic significance of location and type of myocardial infarction:independent adverse outcome associated with anterior location. J Am Coll Cardiol 1988;11(3):453-63.

[19] Ahnve S, Gilpin E, Henning H, et al. Limitations and advantages of ejection fraction for defining high risk after AMI. AM J Cardiol 1986;58(10):872-8.

[20] Toth C, Csomos M, Vadnay I. Significance of early echocardiography in acute myocardial infarct. Orv Hetil 1997;138(13):787-91.

[21] Horowitz RS, Morganorth J. Immediate detection of early high-risk patients with acute myocardial infarction using two dimensional echocardiographic evaluation of left ventricular regional wall motion abnormalities. Am Heart J 1982;103(5):814-22.

[22] Gibson RS, Bishop HL, Stamm RB, et al. Value of early two dimensional echocardiography in patients with acute myocardial infarction. Am J Cardiol 1982;49(5):1110-9. 
[23] The Multicenter Post-infarction Research Group. Risk stratification and survival after myocardial infarction. N Engl J Med 1983;309(6):331-6.

[24] Rouleau JL, Talajic M, Sussex B, et al. Myocardial infraction patients in the 1990s---their risk factors, stratification and survival in Canada:the Canadian Assessment of Myocardial Infraction (CAMI) study. J Am Coll Cardiol 1996;27(5):1119-27.
[25] Finegold JA, Asaria P, Francis DP. Mortality from ischemic heart diseases by country, region and age:statistics from World Health Organisation and United Nations. Int J Cardiol 2013;168(2):934-45.

[26] Kam R, Cutter J, Chew SK, et al. Gender differences in outcome after an acute myocardial infarction in Singapore. Singapore Med J 2002;43(5):243-8. 